\title{
Supporting Clinicians to Use Technology to Deliver Highly Personalized and Measurement-Based Mental Health Care to Young People: Protocol for an Evaluation Study
}

Henriette C Dohnt ${ }^{*}$, MPsych (Clin), BSc; Mitchell J Dowling ${ }^{*}, \mathrm{PhD}$ (ClinPsych); Tracey A Davenport, eMBA, BA (Hons); Grace Lee, MHM, MPH; Shane P Cross, PhD, MPsych (Clin); Elizabeth M Scott, FRANZCP; Yun Ju C Song, PhD; Blake Hamilton, PhD; Samuel J Hockey, MBMSc; Cathrin Rohleder, PhD; Haley M LaMonica, ABPP-CN, $\mathrm{PhD}$, MA, BSc; Ian B Hickie, FASSA, FRANZCP, MD, AM

Brain and Mind Centre, University of Sydney, Camperdown, Australia

*these authors contributed equally

Corresponding Author:

Mitchell J Dowling, PhD (ClinPsych)

Brain and Mind Centre

University of Sydney

94 Mallett St

Camperdown, 2050

Australia

Phone: 610291144100

Email: mitch.dowling@sydney.edu.au

\begin{abstract}
Background: Australia's mental health care system has long been fragmented and under-resourced, with services falling well short of demand. In response, the World Economic Forum has recently called for the rapid deployment of smarter, digitally enhanced health services to facilitate effective care coordination and address issues of demand. The University of Sydney's Brain and Mind Centre (BMC) has developed an innovative digital health solution that incorporates 2 components: a highly personalized and measurement-based (data-driven) model of youth mental health care and a health information technology (HIT) registered on the Australian Register of Therapeutic Goods. Importantly, research into implementation of such solutions considers education and training of clinicians to be essential to adoption and optimization of use in standard clinical practice. The BMC's Youth Mental Health and Technology Program has subsequently developed a comprehensive education and training program to accompany implementation of the digital health solution.
\end{abstract}

Objective: This paper describes the protocol for an evaluation study to assess the effectiveness of the education and training program on the adoption and optimization of use of the digital health solution in service delivery. It also describes the proposed tools to assess the impact of training on knowledge and skills of mental health clinicians.

Methods: The evaluation study will use the Kirkpatrick Evaluation Model as a framework with 4 levels of analysis: Reaction (to education and training), Learning (knowledge acquired), Behavior (practice change), and Results (client outcomes). Quantitative and qualitative data will be collected using a variety of tools, including evaluation forms, pre- and postknowledge questionnaires, skill development and behavior change scales, as well as a real-time clinical practice audit.

Results: This project is funded by philanthropic funding from Future Generation Global. Ethics approval has been granted via Sydney Local Health District's Human Research Ethics Committee. At the time of this publication, clinicians and their services were being recruited to this study. The first results are expected to be submitted for publication in 2021.

Conclusions: The education and training program teaches clinicians the necessary knowledge and skills to assess, monitor, and manage complex needs; mood and psychotic syndromes; and trajectories of youth mental ill-health using a HIT that facilitates a highly personalized and measurement-based model of care. The digital health solution may therefore guide clinicians to help young people recover low functioning associated with subthreshold diagnostic presentations and prevent progression to more serious mental ill-health.

International Registered Report Identifier (IRRID): PRR1-10.2196/24697 
(JMIR Res Protoc 2021;10(6):e24697) doi: 10.2196/24697

\section{KEYWORDS}

mental health service delivery; youth mental health; model of care coordination; transdiagnostic; health information technology; education; training; adoption into clinical practice; Kirkpatrick evaluation

\section{Introduction}

Australia's mental health care system has long been fragmented and under-resourced, with services falling well short of demand [1]. Mental health consumers often receive inconsistent and sporadic treatment delivered by independent services, with little collaborative decision making or multidisciplinary continuous care [2]. At particular risk are young people who do not respond to brief interventions and are unable to access continuous care [3], and those who deteriorate under care [4-6]. Coordination between crisis and ongoing care providers is also poor, with medical staff in emergency departments expressing clear learning needs in developing care plans for patients presenting with mental health problems [7]. The advent of a novel coronavirus and its resulting global COVID-19 pandemic has added additional strain to Australia's mental health services with increasing demands for crisis support and intervention [8-11], highlighting the need for a revolution of current mental health service delivery [12].

The World Economic Forum has recently called for the "...rapid deployment of smarter, digitally-enhanced health services..." as a means of potentially addressing demand issues and facilitating effective care coordination [13]. The recent Australian Productivity Commission's inquiry into mental health also recommends that technology should play a larger role by improving access to the right services at the right time [14]. Moreover, it has been proposed that digital solutions may be a viable method of delivering personalized and measurement-based care to young people, transcending the often-narrow focus on symptom or risk reduction [15]. While there are ethical, data security, and privacy challenges that must be addressed in relation to the implementation of technology-based solutions [13], it has been noted that staff resistance to change and perceived technological complexity frequently present as barriers to the uptake of health information technologies (HITs) [16]. Consequently, education and training that foster engagement and address the use of HITs in practice are essential for successful adoption and optimization of technology-enabled solutions in clinical practice [17]. This increasing need for training and upskilling of clinicians working in digitally enhanced mental health services has not been addressed in the existing literature, which has previously been limited to training specific to clinicians' disciplines, treating specific diagnoses, or intervention modalities [18-20]. As such, there is an urgent need for education and training in the effective use of digital health solutions to meet the increase in demand for mental health services and facilitate effective care coordination across a multidisciplinary workforce.

Researchers at The University of Sydney's Brain and Mind Centre (BMC) have developed the Youth Mental Health (YMH) and Technology Program, which adopts an innovative digital health solution to prevent further progression of mental ill-health for young people in care [21]. This digital health solution includes two key components. The first is the BMC Youth Model, a highly personalized (ie, treatment targeted to address individual needs) and measurement-based (ie, data-driven) model of care generated from over 10 years of longitudinal research led by the BMC to assess multiple clinical and functional domains in young people presenting for mental health care and treatment $[22,23]$. This cohort includes 6743 young people aged 12 to 30 years, which was analyzed to uncover initial underlying neurobiology of mental ill-health in young people [24]. Findings were reported through a supplement of research articles that explore the associations between the multiple clinical and functional domains, neurobiological measures, as well as clinical, social, and functional outcomes [22]. The BMC Youth Model explicitly aims to prevent the progression of emerging and mixed syndromes into more complex and severe forms of illness and to facilitate symptomatic and functional recovery. The second is an HIT such as the InnoWell Platform, which is listed on the Australian Register of Therapeutic Goods - that is a customizable digital toolkit to assist assessment, monitoring, and management of mental ill-health and maintenance of well-being by collecting, storing, scoring, and reporting personal and health information back to consumers and their health professionals to promote collaborative care partnerships [25]. Though we reference the InnoWell Platform as an exemplar HIT, it is important to note that the BMC Youth Model can be facilitated via any HIT so long as its design has been guided by similar clinical and scientific concepts to provide highly personalized and measurement-based care.

The education and training program has been designed to encourage the adoption of highly personalized and measurement-based care in clinical practice, to ensure young people get the right treatment at their first point of entry into care. Specifically, the program focuses on teaching youth mental health professionals how to assess, monitor, and manage complex needs and illness pathways. The education and training program now delivers information via multimodal tools, including online seminars, case study webinars, and in-service workshops. The HIT is referred to throughout the training, as it aids in facilitating highly personalized and measurement-based care (including assessment of clinical stage, pathophysiological mechanisms, and multidimensional needs) and supports the choice of treatment options while tracking a young person's progress.

This paper briefly describes the education and training program and outlines a protocol for an evaluation study to assess the effectiveness of education and training on the adoption and optimization of use of the digital health solution in service delivery. 


\section{Methods}

\section{Education and Training Program}

The education and training program has been designed to be accessed in-person or online. This has been done to provide access to standardized, high-quality training delivered by experienced clinician-researchers from the BMC. This is particularly important for reaching out to those services that would not ordinarily have access to such opportunities, such as health services in regional Australia. The program is currently comprised of online seminars, case study webinars, and in-service training workshops.

The online seminars introduce the theoretical, clinical, and scientific underpinnings of the YMH and Technology Program that provide the background knowledge necessary for clinicians to understand the digital health solution and begin to apply it to their own clinical practice. To date, 6 seminars delivered by experienced clinician researchers (authors ES and IH) have been recorded covering approximately 60 minutes each (see Textbox 1) and are freely accessible through the BMC Youth Mental Health Research YouTube Channel.

Textbox 1. Modules of the education and training program.

These online seminars are further enhanced by case study webinars, which detail practical application of the digital health solution and help to translate newly acquired knowledge into clinical skills [26]. Two such case study webinars spanning an hour each have been recorded to date and cover 3 cases, their initial presentation, and the tracking of their syndromes over time demonstrating the value of the digital health solution.

In-service training workshops will be delivered by BMC clinician-researchers and focus on the practical application of the BMC Youth Model into the specific youth mental health service. The workshops typically include case studies common to the participating service. These workshops can be conducted either face-to-face or online. To date, 2 regional headspace services in Northern New South Wales have attended in-service training workshops.

In order to encourage and reward participation in all components of the education and training program, continuing professional development points are available for psychiatrists, psychologists, mental health nurse practitioners, social workers, and occupational therapists. To further encourage engagement, additional specialized supplemental webinars (eg, applying the BMC Youth Model to understand the relationship between circadian rhythms and mental health and applying the BMC
Youth Model to enhance psychological therapy for individuals with anxiety and other mood disorders) and case studies will be released, demonstrating how the BMC Youth Model can be applied to specific disorders and complex cases.

\section{An Evaluation Framework}

The importance of a standardized and consistent framework for evaluation of education and training programs has previously been highlighted [27,28]. Evaluations often only focus on participants' reactions and acquired knowledge and tend to neglect the assessment of behavioral change or impact on client outcomes, which are both critical factors in determining effectiveness [27].

This study will utilize the Kirkpatrick Evaluation Model, which includes 4 levels of training evaluation including: Reaction, Learning, Behavior, and Results [29] (see Table 1). This evaluation framework is ideally suited to a mixed methods approach combining quantitative and qualitative methods, as each level of evaluation requires the implementation of appropriate methodology that is complementary to, but independent of, the other levels. Aspects of the Kirkpatrick Evaluation model have been successfully used in mental health training, for instance in suicide prevention [30].

Table 1. Applying the Kirkpatrick Evaluation Model as an evaluation framework for the education and training program.

\begin{tabular}{ll}
\hline Level & Key question \\
\hline Level 1: Reaction & What are the reactions of clinicians to the digital health solution? \\
Level 2: Learning & Have clinicians learned the relevant knowledge relating to the digital health solution? \\
Level 3: Behavior & Have clinicians transferred and applied their knowledge of the digital health solution to standard clinical practice? \\
Level 4: Results & $\begin{array}{l}\text { What has been the impact of the digital health solution on service performance, such as efficiency, clinical safety, } \\
\text { and clinical outcomes? }\end{array}$ \\
\hline
\end{tabular}

The Kirkpatrick Evaluation Model [29] also highlights the importance of ongoing education and training, as the majority of learning is acquired through practice. In order to sustain education and training, the Kirkpatrick model encourages 
monitoring, reinforcing, encouraging, and rewarding. For this study, monitoring and reinforcing will be completed at 3 months, 6 months, 9 months, and 12 months post-initial education and training, with individual progress reports being provided in order to encourage and reward their adoption of the YMH and Technology Program.

\section{Measures}

\section{Participant Profile}

Before commencing the education and training program, clinicians will be asked to complete a participant profile. This profile includes questions regarding their educational background, professional background, years of clinical experience, and reasons for participating in the education and training program.

\section{Online Seminar, Case Study Webinar, In-Service Workshop Evaluation Form}

This form assesses clinician reaction (Level 1) to the education and training program, including satisfaction, engagement, and relevance. This form consists of 20 items, of which the first 15 are rated on a 5 - point Likert scale ranging from " 1 = totally disagree" to " $5=$ totally agree," and the remaining 5 questions represent an open-ended format. Clinician satisfaction will also be measured using the Training Satisfaction Rating Scale, an existing scale with good content validity [31]. This scale is a 12-item questionnaire assessing participant agreement to objectives and content (eg, "In my opinion, the planned objectives of the [online seminar/ case study webinar/ in-service workshop] were met."), method and training context (eg, "The [online seminar/ case study webinar/ in-service workshop] enabled us to take an active part in training."), and usefulness and overall rating (eg, "The education and training received is useful for my personal development."). In addition to the existing scale, 3 items will be used to evaluate the quality of facilitation (eg, "The facilitator(s) were knowledgeable about the education and training topics."). Lastly, participants will be invited to provide qualitative feedback on the education and training program regarding most liked and disliked aspects, major learnings, and expected results of the training (eg, "What aspects of the training could be improved?").

\section{Knowledge Questionnaire}

This questionnaire assesses clinician learning (Level 2) of key concepts of the digital health solution through the online seminars. This questionnaire consists of 30 multiple-choice questions relating to multidimensional assessment and outcomes, clinical staging and illness trajectories, social and biological development, real-time monitoring, and technology-enabled practice. Clinicians must select correct answers out of a list of answers that includes equally plausible distractors. In order to keep the questionnaire brief and reduce the chances of participants learning the answers through repetition, clinicians will be asked to complete 5 randomly selected questions at each time point (ie, at baseline, 3 months, 6 months, 9 months, and 12 months). Furthermore, at each time point, participants will be asked to identify which webinars and case studies they have watched in order to be able to link training engagement with outcomes.

\section{Skill Development Scale}

This scale assesses confidence in clinical skills (Level 3) central to the BMC Youth Model using 8 visual analogue scales coded as values "0" to "100." Marks to the left represent low confidence in development of skills, and marks to the right indicate high confidence in development of skills. The 8 items relate to multidimensional assessment, clinical staging, case formulation and treatment planning, shared decision making, intervention skills, outcome monitoring, and care continuity and coordination. Respondents are asked to reflect on each of these areas and rate how well they feel they perform these tasks in clinical practice.

\section{Behavior Change Scale}

This scale assesses clinician change in behavior (Level 3) using a scale consisting of 9 items that ask how frequently they utilized new knowledge and skills related to multidimensional assessment, clinical staging, intervention matching, shared decision making, monitoring, responding to increased suicidality, and continuity of care. Clinicians rate how often they employed a skill in their standard clinical practice in the past fortnight on a 5-point Likert scale ranging from " 1 = not at all (with no clients)" to " $5=$ always (all of the time, for all clients)." For each item, clinicians can also indicate that the particular skill is not applicable to their service $(0=\mathrm{N} / \mathrm{A})$.

\section{Clinical Practice Audit}

Change in clinician practice (Level 3) and impact on client outcomes (Level 4) will be assessed using real-time and aggregated data from the InnoWell Platform [32]. Indicators of adoption of the digital health solution include, for example, the frequency with which clinicians access the platform and time spent on the platform, accuracy of clinical staging assignment, and the recommendation of treatment options. Indicators of impact on client outcomes include, for example, efficiency (eg, time to first assessment and wait time for clinical intervention), clinical safety (eg, notifications for suicide thoughts and behaviors and average time to respond to notifications), and clinical outcomes (eg, changes over time in psychological distress as well as social and occupational functioning).

\section{Procedure}

The timeline of procedures is summarized in Figure 1. Before commencing the online education and training components, clinicians will complete the participant profile and a baseline knowledge questionnaire, skill development scale, and behavior change scale. A baseline clinical practice audit will also be completed. On completion of the 6 online seminars, the 2 case study webinars, and the in-service workshops, participants will be asked to complete a posttest knowledge questionnaire as well as the skill development and behavior change scales. Importantly, participating clinicians will additionally be contacted after 3 months, 6 months, 9 months, and 12 months and asked to complete the posttest knowledge questionnaire, skill development scale, and behavior change scale as a means to monitor and reinforce knowledge, skills, and behavior [29]. Together with real-time data from the InnoWell Platform (pre-education and training and then every 3 months posteducation and training), participants will privately receive 
a progress report to encourage and reward their adoption and optimization of the digital health solution [29]. Finally, after each online seminar, case-study webinar, or in-service workshop, clinicians will be asked to complete the evaluation form.

Figure 1. Flow chart of evaluation methods pre- and post-education and training.

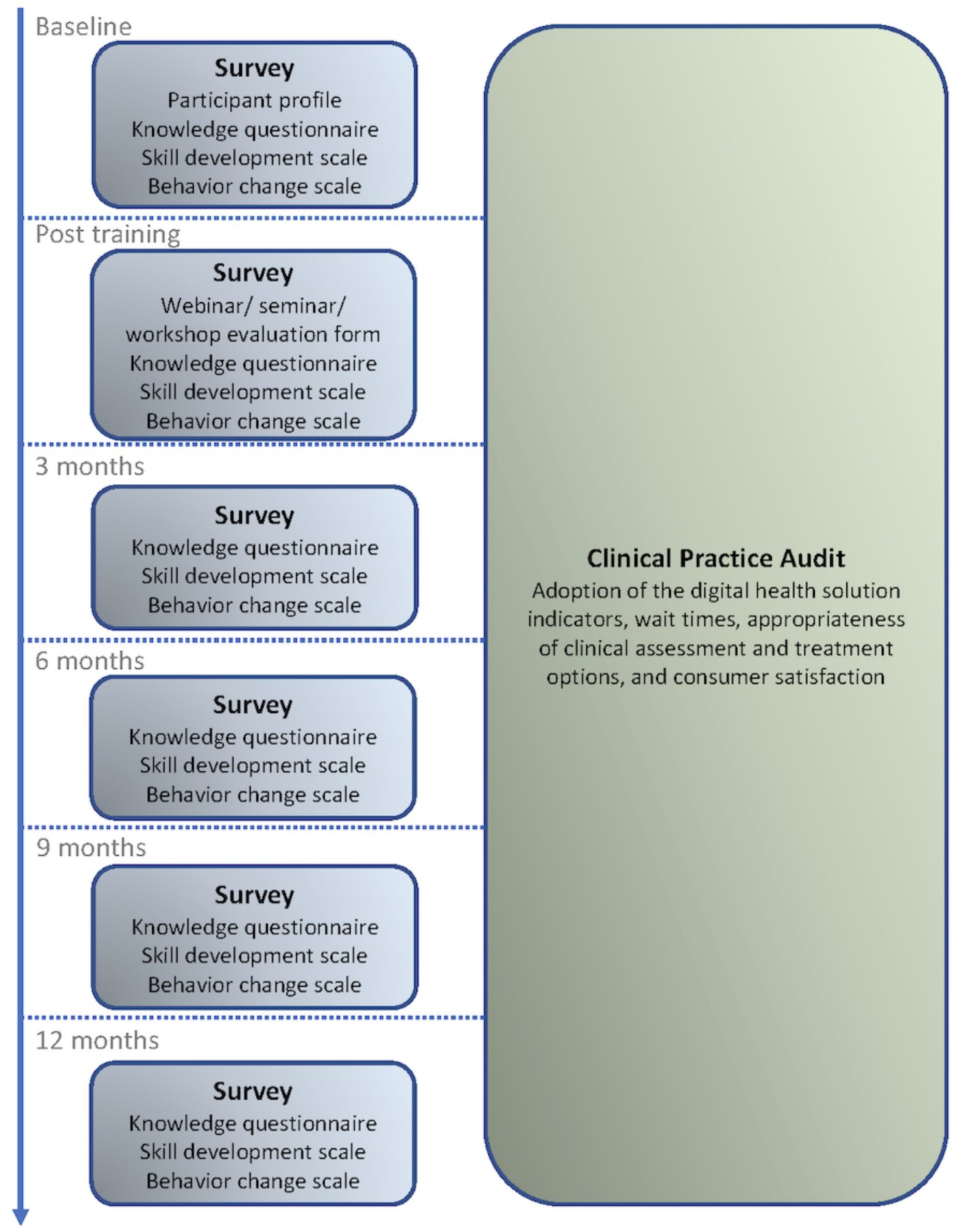

\section{Participants}

Participants will be drawn from 11 participating headspace services in Australia who have previously agreed to implement the HIT. The services include 5 from Sydney, 5 on the North Coast of New South Wales, and 1 in South Australia. Participants will include mental health professionals (eg, psychologists, social workers, mental health nurses, occupational therapists) and service managers. The sample size will be determined by participating services, with all health professionals and service managers being invited to participate in the education and training program.

\section{Statistical Analyses}

In order to assess participants' reactions to the training, a mixed methods approach will be utilized, specifically a Triangulation 
Design, Validating Quantitative Data Model [33]. This design would allow the for the quantitative findings (eg, objectives and content, method and training context, usefulness, and quality of facilitation) to be validated and expanded on by including a few open-ended qualitative questions (eg, most liked and disliked aspects, major learnings, and expected results of the training).

Descriptive statistics will be used to analyze quantitative data gathered from the combined evaluation form data, as well as individually for the online seminars, case study webinars, and in-service workshops. A thematic analysis will be conducted to analyze qualitative data collected via the evaluation forms to identify patterns or themes within the data [34]. Procedurally, thematic analysis entails (1) becoming familiar with the data, (2) generating initial codes, (3) searching for themes, (4) reviewing themes, (5) defining and naming themes, and (6) reporting the final thematic concepts. The data will be coded using NVivo by a minimum of 2 different researchers in order to assess interrater reliability. These resulting thematic concepts can then be used to validate and embellish the quantitative survey findings.

In order to assess Learning, Behavior, and Results, pre- and postsurveys and clinical practice audit data will be compared using paired $t$ tests to understand short-term impacts, and repeated measures analysis of variance will be used to understand long-term changes in knowledge, skill, behavior, and client outcomes.

\section{Ethics and Dissemination}

Ethics approval has been granted via Sydney Local Health District's Human Research Ethics Committee (Protocol No X18-0499 \& HREC/18/RPAH/715). Research findings will be disseminated through peer-reviewed journals and scientific conference presentations. Participant data will be nonidentifiable and aggregated.

\section{Results}

As of September 2020, clinicians and their services were being recruited to this study. Data collection is expected to be completed and the first results to be submitted for publication in 2021. Results will then be disseminated to the participants, the public, and researchers through publications in journals and presentations at conferences.

\section{Discussion}

This paper details a comprehensive protocol for an evaluation study to assess the effectiveness of the education and training program on the adoption and optimization of use of the digital health solution to provide the knowledge and skills to assess, monitor, and manage the complex needs of young people presenting with mental ill-health problems.
Evaluations of education and training programs within mental health generally focus on the perceived usefulness of such programs, increased knowledge, and confidence in using acquired skills, but frequently neglect assessing the impact on changing clinician knowledge, skills, behaviors, and client outcomes [35]. By using the Kirkpatrick Evaluation Model [29], this study will assess the effectiveness of the education and training program in meeting the learning needs of clinicians (Reaction), increasing clinicians' knowledge of youth mental health (Learning), clinicians adopting the digital health solution in standard clinical practice (Behavior), and improving service performance (eg, efficiency, clinical safety, and clinical outcomes; Results).

While the evaluation protocol will produce valuable insights, there are limitations that should be noted. First, the measures of knowledge and skill rely on self-rating, which are subjective and may be influenced by the participant's level of self-awareness [36,37]. Second, it is generally recognized as being quite difficult to objectively measure the competence of mental health practitioners [37]. Last, the Kirkpatrick model is not without its flaws; for example, it has received criticism for being overly simplified and lacking an ability to provide information about how training can be improved [38]. Nevertheless, the Kirkpatrick model has been successfully utilized in prior mental health training [30] and will provide data in a way that can guide further research.

The research protocol presented here is the first of its kind to explore the education and training of mental health professionals in the use and benefits of technology-enabled solutions. As such, it has the potential to have a significant impact on determining how mental health professionals are trained as technology becomes more integrated into their practice $[13,14]$. The quantitative and qualitative data obtained from this study will be used to iteratively adapt and improve the education and training program in order to ensure that young people get the right care the first time and to prevent further progression of mental ill-health while in care. These results will also guide the creation of additional education and training resources to keep participating clinicians engaged and to the ensure sustainability of the digital health solution. Future resources may include a case study workbook and the creation of a community of practice that could provide a shared context for practitioners, enabling dialogue between practitioners who might not ordinarily have the chance to interact [39]. Further, our education and training team is currently exploring a more sophisticated online interactive platform to promote ongoing customized learning with participating youth mental health services.

Ultimately, this research will help teach mental health professionals how to facilitate a highly personalized and measurement-based model of care. This may therefore guide clinicians to help young people recover from low functioning associated with subthreshold diagnostic presentations and prevent progression to more serious mental ill-health. 


\section{Acknowledgments}

This project is an investigator-initiated study and supported by philanthropic funding, for which donor(s) are families affected and wish to remain anonymous. The authors would like to acknowledge Future Generation Global (FGG) for funding the YMH and Technology Program, which aims to improve young people's access to quality mental health care. The authors would also like to thank Ms Tanya Jackson, Ms Sarah Piper, and Ms Alexis Hutcheon for their contributions to the YMH and Technology Program.

\section{Conflicts of Interest}

IH was an inaugural Commissioner on Australia's National Mental Health Commission (2012-18). He is the Co-Director, Health and Policy at the BMC University of Sydney. The BMC operates an early-intervention youth services at Camperdown under contract to headspace. He is the Chief Scientific Advisor to, and a 5\% equity shareholder in, InnoWell Pty Ltd. InnoWell was formed by the University of Sydney (45\% equity) and PwC (Australia; 45\% equity) to deliver the AU \$30-million Australian Government-funded Project Synergy (2017-20; a 3-year program for the transformation of mental health services) and to lead transformation of mental health services internationally through the use of innovative technologies. TD is now Director (Research and Evaluation), Design and Strategy Division, Australian Digital Health Agency. Other authors on this paper have no conflicts of interest to disclose.

TD is now Director (Research and Evaluation), Design and Strategy Division, Australian Digital Health Agency.

Other authors on this paper have no conflicts of interest to disclose.

\section{References}

1. 2014 Contributing Lives Review. Australian Government National Mental Health Commission. 2014. URL: https://www. mentalhealthcommission.gov.au/Monitoring-and-Reporting/national-reports/2014-Contributing-Lives-Review [accessed 2021-05-14]

2. Crowe J. Reform, revolution and disruption in mental health care: a consumer's perspective. Public Health Res Pract 2017 Apr 27;27(2):1 [FREE Full text] [doi: 10.17061/phrp2721711] [Medline: 28474048]

3. Cross SP, Hermens DF, Hickie IB. Treatment patterns and short-term outcomes in an early intervention youth mental health service. Early Interv Psychiatry 2016 Feb 27;10(1):88-97. [doi: 10.1111/eip.12191] [Medline: 25263607]

4. Cross SP, Scott JL, Hermens DF, Hickie IB. Variability in Clinical Outcomes for Youths Treated for Subthreshold Severe Mental Disorders at an Early Intervention Service. Psychiatr Serv 2018 May 01;69(5):555-561. [doi:

10.1176/appi.ps.201700046] [Medline: 29334880]

5. Hickie IB, Hermens DF, Naismith SL, Guastella AJ, Glozier N, Scott J, et al. Evaluating differential developmental trajectories to adolescent-onset mood and psychotic disorders. BMC Psychiatry 2013 Nov 12;13(1):1. [doi: $\underline{10.1186 / 1471-244 x-13-303]}$

6. Iorfino F, Hermens DF, Cross SP, Zmicerevska N, Nichles A, Badcock C, et al. Delineating the trajectories of social and occupational functioning of young people attending early intervention mental health services in Australia: a longitudinal study. BMJ Open 2018 Mar 27;8(3):e020678 [FREE Full text] [doi: 10.1136/bmjopen-2017-020678] [Medline: 29588325]

7. Sivakumar S, Weiland TJ, Gerdtz MF, Knott J, Jelinek GA. Mental health-related learning needs of clinicians working in Australian emergency departments: a national survey of self-reported confidence and knowledge. Emerg Med Australas 2011 Dec;23(6):697-711. [doi: 10.1111/j.1742-6723.2011.01472.x] [Medline: 22151668]

8. Hickie I, Atkinson J, Rock D, Millard M, Cross S, Scott L, et al. "No more waitlists" webinar - digiHealth solutions for better mental health service delivery. University of Sydney. 2020 May 12. URL: https://www.youtube.com/ watch?v=YPZJzuDpFNQ [accessed 2021-05-14]

9. Holmes EA, O'Connor RC, Perry VH, Tracey I, Wessely S, Arseneault L, et al. Multidisciplinary research priorities for the COVID-19 pandemic: a call for action for mental health science. Lancet Psychiatry 2020 Jun;7(6):547-560 [FREE Full text] [doi: 10.1016/S2215-0366(20)30168-1] [Medline: 32304649]

10. Russell L. Covid-19's second wave. Inside Story. 2020 May 26. URL: https://insidestory.org.au/covid-19s-second-wave/ [accessed 2021-05-14]

11. Stayner T. Australia's suicide rate could surge due to economic fallout of coronavirus. SBS News. 2020 May 07. URL: https://www.sbs.com.au/news/australia-s-suicide-rate-could-surge-due-to-economic-fallout-of-coronavirus [accessed 2021-05-14]

12. Davenport TA, Cheng VWS, Iorfino F, Hamilton B, Castaldi E, Burton A, et al. Flip the Clinic: A Digital Health Approach to Youth Mental Health Service Delivery During the COVID-19 Pandemic and Beyond. JMIR Ment Health 2020 Dec 15;7(12):e24578 [FREE Full text] [doi: 10.2196/24578] [Medline: 33206051]

13. Empowering 8 Billion Minds: Enabling Better Mental Health for All via the Ethical Adoption of Technologies. World Economic Forum. 2019 Jul 1. URL: https://www.weforum.org/whitepapers/ empowering-8-billion-minds-enabling-better-mental-health-for-all-via-the-ethical-adoption-of-technologies [accessed 2021-05-14] 
14. Mental health inquiry report. Australian Government Productivity Commission. URL: https://www.pc.gov.au/inquiries/ completed/mental-health/report [accessed 2021-05-14]

15. Iorfino F, Cross SP, Davenport T, Carpenter JS, Scott E, Shiran S, et al. A Digital Platform Designed for Youth Mental Health Services to Deliver Personalized and Measurement-Based Care. Front Psychiatry 2019 Aug 23;10:595 [FREE Full text] [doi: 10.3389/fpsyt.2019.00595] [Medline: 31507465$]$

16. Wendland J, Lunardi GL, Dolci DB. Adoption of health information technology in the mobile emergency care service. RAUSP 2019 Jul 08;54(3):287-304. [doi: 10.1108/RAUSP-07-2018-0058]

17. Yen P, McAlearney AS, Sieck CJ, Hefner JL, Huerta TR. Health Information Technology (HIT) Adaptation: Refocusing on the Journey to Successful HIT Implementation. JMIR Med Inform 2017 Sep 07;5(3):e28 [FREE Full text] [doi: 10.2196/medinform.7476] [Medline: 28882812]

18. Birrane J, Swan D, Aherne D, Davis R, Hannigan A, McPhillips D, et al. Development and evaluation of an educational intervention in youth mental health for primary care practitioners. Ir J Psychol Med 2015 Mar 22;32(1):137-146. [doi: 10.1017/ipm.2014.71] [Medline: 30185272]

19. Carmel A, Logvinenko E, Valenti ES. Evaluation of a Dialectical Behavior Therapy Psychiatry Residency Training Program. Acad Psychiatry 2019 Feb 12;43(1):37-40. [doi: 10.1007/s40596-018-0993-4] [Medline: $\underline{\text { 30315500] }}$

20. Macneil C, Foster F, Nicoll A, Osman H, Monfries R, Cotton S. Evaluation of a professional development training programme for mental health clinicians specializing in early psychosis. Early Interv Psychiatry 2018 Jun 26;12(3):483-490. [doi: 10.1111/eip.12424] [Medline: 28744970]

21. Piper S, Davenport TA, LaMonica H, Ottavio A, Iorfino F, Cheng VWS, et al. Implementing a digital health model of care in Australian youth mental health services: protocol for impact evaluation. BMC Health Serv Res 2021 May 12;21(1):452 [FREE Full text] [doi: 10.1186/s12913-021-06394-4] [Medline: $\underline{\text { 33980229] }}$

22. Hickie IB, Scott EM, Cross SP, Iorfino F, Davenport TA, Guastella AJ, et al. Right care, first time: a highly personalised and measurement-based care model to manage youth mental health. Med J Aust 2019 Nov 03;211 Suppl 9(S9):S3-S46. [doi: 10.5694/mja2.50383] [Medline: $\underline{31679171]}$

23. Carpenter JS, Iorfino F, Cross S, Nichles A, Zmicerevska N, Crouse JJ, et al. Cohort profile: the Brain and Mind Centre cohort: tracking multidimensional outcomes in young people presenting for mental healthcare. BMJ Open 2020 Mar 29;10(3):e030985 [FREE Full text] [doi: 10.1136/bmjopen-2019-030985] [Medline: 32229519]

24. Carpenter JS, Iorfino F, Cross S, Nichles A, Zmicerevska N, Crouse JJ, et al. Cohort profile: the Brain and Mind Centre cohort: tracking multidimensional outcomes in young people presenting for mental healthcare. BMJ Open 2020 Mar 29;10(3):e030985 [FREE Full text] [doi: 10.1136/bmjopen-2019-030985] [Medline: 32229519]

25. Davenport TA, LaMonica HM, Whittle L, English A, Iorfino F, Cross S, et al. Validation of the InnoWell Platform: Protocol for a Clinical Trial. JMIR Res Protoc 2019 May 31;8(5):e13955 [FREE Full text] [doi: 10.2196/13955] [Medline: 31152524]

26. McLean SF. Case-Based Learning and its Application in Medical and Health-Care Fields: A Review of Worldwide Literature. J Med Educ Curric Dev 2016;3:1 [FREE Full text] [doi: 10.4137/JMECD.S20377] [Medline: 29349306]

27. Dubrowski A, Morin M. Evaluating pain education programs: an integrated approach. Pain Res Manag 2011;16(6):407-410 [FREE Full text] [doi: 10.1155/2011/320617] [Medline: 22184548]

28. Santos M, Vicente T, Monteiro A. Temporalities in evaluation of training courses: standards and restrictions practiced by human resources professionals. Work 2012;41(2):217-226. [doi: 10.3233/WOR-2012-1290] [Medline: 22297785]

29. Kirkpatrick JD, Kirkpatrick WK. Kirkpatrick's Four Levels of Training Evaluation. Alexandria, VA: ATD Press; 2016.

30. Sale E, Hendricks M, Weil V, Miller C, Perkins S, McCudden S. Counseling on Access to Lethal Means (CALM): An Evaluation of a Suicide Prevention Means Restriction Training Program for Mental Health Providers. Community Ment Health J 2018 Apr 28;54(3):293-301. [doi: 10.1007/s10597-017-0190-z] [Medline: 29185154]

31. Holgado Tello FP, Chacón Moscoso S, Barbero García I, Sanduvete Chaves S. Training Satisfaction Rating Scale. European Journal of Psychological Assessment 2006 Jan;22(4):268-279. [doi: 10.1027/1015-5759.22.4.268]

32. Hickie IB, Davenport TA, Burns JM, Milton AC, Ospina-Pinillos L, Whittle L, et al. Project Synergy: co-designing technology-enabled solutions for Australian mental health services reform. Med J Aust 2019 Oct 06;211 Suppl 7(S7):S36-S39. [doi: $\underline{10.5694 / \mathrm{mja} 2.50349]}$ [Medline: $\underline{31587276}$ ]

33. Creswell JW, Clark VLP, editors. Introducing a mixed method design. In: Designing and conducting mixed methods research. Thousand Oaks, CA: Sage Publications, Inc; 2007:58-88.

34. Braun V, Clarke V. Using thematic analysis in psychology. Qualitative Research in Psychology 2006 Jan;3(2):77-101. [doi: 10.1191/1478088706qp063oa]

35. Sinclair PM, Kable A, Levett-Jones T, Booth D. The effectiveness of Internet-based e-learning on clinician behaviour and patient outcomes: A systematic review. Int J Nurs Stud 2016 May;57:70-81. [doi: 10.1016/j.ijnurstu.2016.01.011] [Medline: 27045566]

36. McManus F, Rakovshik S, Kennerley H, Fennell M, Westbrook D. An investigation of the accuracy of therapists' self-assessment of cognitive-behaviour therapy skills. Br J Clin Psychol 2012 Sep;51(3):292-306 [FREE Full text] [doi: 10.1111/j.2044-8260.2011.02028.x] [Medline: 22803936]

37. Waltman S, Frankel SA, Williston MA. Improving clinician self-awareness and increasing accurate representation of clinical competencies. Practice Innovations 2016 Sep;1(3):178-188 [FREE Full text] [doi: 10.1037/pri0000026] 
38. Bates R. A critical analysis of evaluation practice: the Kirkpatrick model and the principle of beneficence. Evaluation and Program Planning 2004 Aug;27(3):341-347. [doi: 10.1016/j.evalprogplan.2004.04.011]

39. Barnett S, Jones SC, Bennett S, Iverson D, Bonney A. General practice training and virtual communities of practice - a review of the literature. BMC Fam Pract 2012 Aug 21;13(1):87 [FREE Full text] [doi: 10.1186/1471-2296-13-87] [Medline: 22905827]

\section{Abbreviations \\ BMC: Brain and Mind Centre \\ HIT: health information technology \\ YMH: Youth Mental Health}

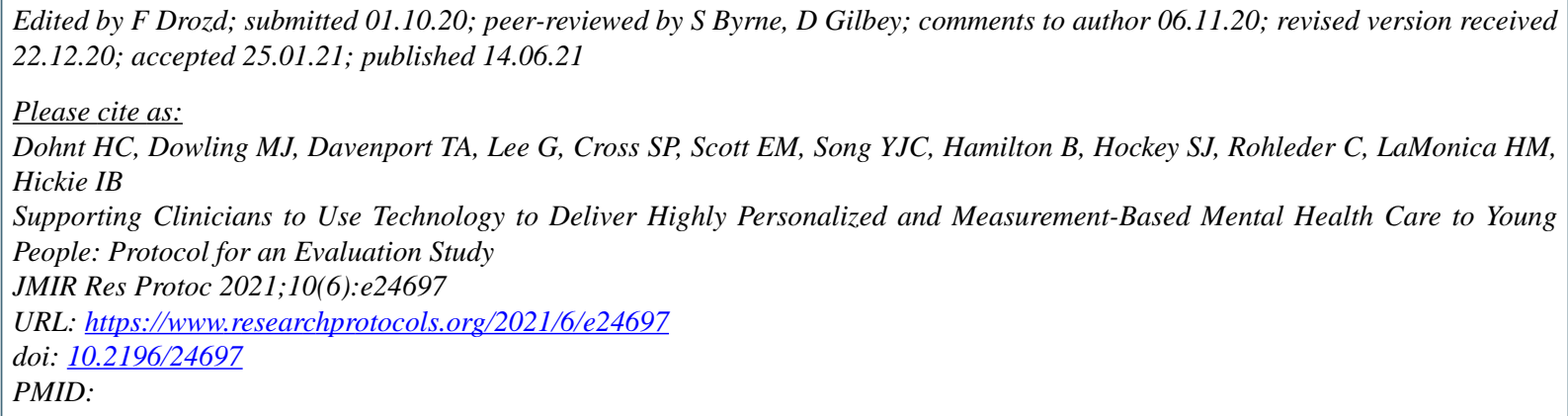

CHenriette C Dohnt, Mitchell J Dowling, Tracey A Davenport, Grace Lee, Shane P Cross, Elizabeth M Scott, Yun Ju C Song, Blake Hamilton, Samuel J Hockey, Cathrin Rohleder, Haley M LaMonica, Ian B Hickie. Originally published in JMIR Research Protocols (https://www.researchprotocols.org), 14.06.2021. This is an open-access article distributed under the terms of the Creative Commons Attribution License (https://creativecommons.org/licenses/by/4.0/), which permits unrestricted use, distribution, and reproduction in any medium, provided the original work, first published in JMIR Research Protocols, is properly cited. The complete bibliographic information, a link to the original publication on https:/www.researchprotocols.org, as well as this copyright and license information must be included. 